\title{
A Cross Layer Fast Handover Scheme in VANET
}

\author{
Kuan-Lin Chiu \\ Department of Computer Science \\ and Information Engineering \\ National Chung Cheng University \\ Chiayi 621, Taiwan, R. O. C. \\ chiukl@cs.ccu.edu.tw
}

\author{
Ren-Hung Hwang \\ Department of Computer Science \\ and Information Engineering \\ National Chung Cheng University \\ Chiayi 621, Taiwan, R. O. C. \\ rhhwang@cs.ccu.edu.tw
}

\author{
Yuh-Shyan Chen \\ Department of Computer Science \\ and Information Engineering \\ National Taipei University \\ Taiwan, R.O.C. \\ yschen@csie.ntpu.edu.tw
}

\begin{abstract}
This study presents a cross-layer fast handover scheme for VANET, called vehicular fast handover scheme (VFHS), where the physical layer information is shared with the MAC layer, to reduce the handover delay. The key idea of VFHS is to utilize oncoming side vehicles (OSVs) to collect physical and MAC layers information of passing through RVs and broadcast the information to vehicles that are temporarily disconnected, referred to as broken vehicles (BVs). A BV can thus perform a rapid handover when it enters the transmission range of the approaching RVs. The effectiveness of VFHS is verified using ns2 simulations. Simulation results indicate that VFHS significantly decreases handover latency and packet loss.
\end{abstract}

\section{INTRODUCTION}

Intelligent Transport System (ITS) [7] has been proposed to improve vehicle safely and comfort of drivers and passengers using large scale wireless techniques, e.g. Vehicular Ad Hoc Network (VANET) [11]. Considering the future transportation environment, a promising way to access the Internet is to use multihop transmission technique, e.g. IEEE 802.16j Worldwide Interoperability for Microwave Access Mobile Multihop Relay (WiMAX MMR). As proposed in [6], special types of vehicles, such as public transportation bus, are good candidates to serve as relay nodes, referred to as relay vehicles (RV). An RV provides communication and mobility management services to its neighboring vehicles in the way that neighboring vehicles transmit packets to the Internet through the RV. An RV together with its neighboring vehicles form a cluster network. The multihop environment is particular suitable for vehicles in freeways because vehicles driving in the same direction are moving with nearly the same speed. For example, in Taiwan, according to statistics measured during year 2007 [2], there are 54,347,929 coaches and 475,502,719 sedans on the freeway, respectively. That is, on average, a coach is surrounded by 8.7 sedans. Another statistic shows that, on average, a coach passes though a toll booth every 5.3 seconds. Therefore, a coach equipped with WiMAX relay node which provides connectivity for 8 or 9 sedan is in a promising solution for VANET on freeways in Taiwan. On the other hand, dynamics of coach distribution on the freeway, a sedan still needs to face the dis-connectivity problem when it is out of the transmission range of any coaches and access points. The sedan hence needs to scan all channels to search next access point. Therefore, this work aims to solve disconnectivity problem by improving L2handover procedure in this kind of VANET.

In this paper, a cross layer fast handover scheme, termed vehicular fast handover scheme (VFHS), is proposed. VFHS is a hybrid method and improves the layer 2 handover performance via utilizing topology information broadcasted by oncoming small size vehicles (OSV). An OSV is able to collect the physical information of an RV via performing the entry procedure or receiving the RV's network advertisement message. The OSV uses the set of predefined channel frequencies to broadcast network topology message (NTM) to BVs. With NTM, the unconnected vehicle is able to know which channel to listen to when it enters the transmission range of the RV in front. As a consequence, the handover latency could be significantly reduced.

The rest of this article is organized as follow. Section II discusses related works. The details of the VFHS scheme and our argument of the context-aware are described in section III. The performance analysis is presented in Section IV. Finally, section V summarizes this work.

\section{RELATED WORK}

Numerous improved handover schemes have been presented recently. All of them concentrate on pure, standard mobile WiMAX networks. This section describes and summarizes these improved schemes.

Several existing schemes for reducing handover latency in WiMAX are categorized to three categories: improving scanning latency [3][4][10], inter-cell handover limitation [9] and cross layer design [5]. A prediction-based pre-coordination mechanism (PCM) has been presented to support fast handover in WiMAX network by improving the scanning latency. The PCM is practiced on mobile WiMAX, which adopts the position information of the MS, e.g. location, distance, and direction, to predict the target BS. The ACS [10] focuses on to selecting a minimum scanning period for MSs. During handover, the serving BS estimates the scanning time of each neighbor BS, and notifies this information to the MS. The MS chooses the neighbor with the shortest scanning period as its target BS. Rouil et al. studied two strategies, namely Most Recently Used Strategy (MRU) and Most Frequently Used 
Strategy (MFU), to decrease scanning times in MSs based on the historical channel information of neighboring BSs. In inter-cell handover limitation, Park et al. [9] developed a cellID-based handover method in an MMR network to lower the handover overhead. The cell-ID scheme modifies the standard BSID format to support hierarchical BS/RS ID format. Finally, Chen et al. [5] proposed a cross layer design fast handover scheme to eliminate the handover latency using MAC message exchanging. Unfortunately, previous studies do not consider the characteristics of the underlying VANETs, namely storecarry-forward routing, high-speed mobility, and continuous transition between RVs.

\section{VFHS SCHEME IN VANET NETWORK}

In VFHS, the cross-layer design is considered to provide cross layer topology message for the BV. The description of the VFHS scheme is formally illustrated in this section.

\section{A. System Model}

The system model of VFHS on freeways is shown in Fig. 1.In this model, speeds of vehicles are unlimited as long as their relative speed could be supported by the MMR WiMAX network. We also assume that each vehicle is equipped with a location sensor, such as Global Positioning System (GPS), such that it is aware of its position. There are three kinds of vehicle considered in our model which are described as follows:

- Relay Vehicle (RV): An RV is a large vehicle that can provide the capability of relay and mobile management of the MMR WiMAX network to its neighboring vehicles. A $\mathrm{RV}$ together with vehicles in its transmission range forms a cluster network. The transmission range is denoted in by circles.

- Broken Vehicle (BV): A BV is a small vehicle that is outside of the coverage of any RV, and needs to transmit packets.

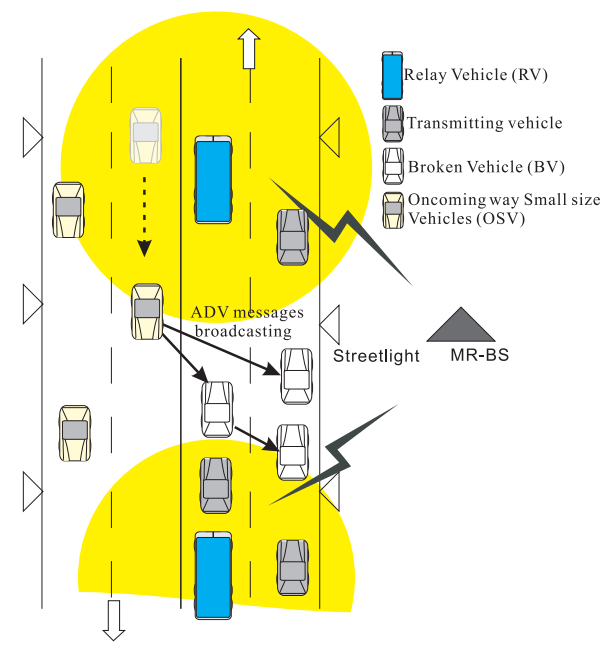

Fig. 1. System Model

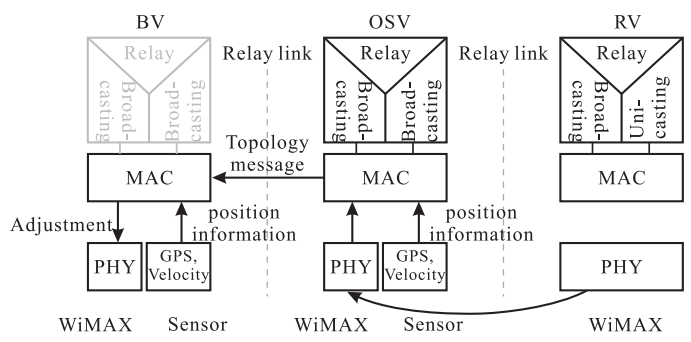

Fig. 2. Cross layer stack integration position information with WiMAX MAC layer

- Oncoming way Small size Vehicle (OSV): An OSV is a small vehicle driving on the oncoming direction, and has no packets to transmit. The OSV can accumulate the physical layer information of the RV located in the opposite direction (oncoming RV), and provide this information to BVs with the cross-layer network topology message (NTM) for facilitating fast handover. The OSV uses a set of specific channels to broadcast NTM to BVs.

\section{B. Cross Layer Concept on VFHS}

To support dynamic adaptation, VFHS adopts explicit crosslayer design [8][12] to provide signaling message to cross MAC and physical layers. Figure 2 illustrates the cross-layer structure of the system under consideration. NTM is a crosslayer message comprising physical (position and channel) and MAC (WiMAX) layers. In VFHS, the position and channel information of the oncoming RV are accumulated, abstracted and managed by the OSV when acquires. In addition to the RV information, the OSV inserts its position information into NTM, and broadcasts to BVs. Upon receiving NTM, BVs can adjust the setting of the channel frequency of the WiMAX adapter immediately by comparing its location for synchronizing with the target RV; instead of scanning all channel frequencies sequentially by physical layer. Therefore, according to this cross-layer design, VFHS reduces the time of scanning procedure.

\section{Packet Format}

We further define a message format termed topology message which the OSV maintains. Recall that the message is broadcast by an OSV and carries physical layer information of an oncoming RV. Description of each field is given as follows. The first 32-bit identifies an RV which consists of two components: a 24-bit MR-BS id (MRBSID) and a 8-bit RV id (RVID). The rationale is that each MR-BS will serve several RVs that are in its transmission range. Therefore, it is a twolevel hierarchical address. In addition, direction information of an RV is also encoded in the (RVID). The next two fields, RV Speed and OSV Speed, represent the velocities of the RV and OSV, repectively. They could be estimated via GPS data or measured by analyzing the REP-REQ/REP-RSP messages. The field Direction $(D)$ encodes the direction of the OSV which can also be calculated based on the GPS data. The Hop Count $(H C)$ value records the number of vehicles (hops) 
that this topoloby message has been traversed. The field Time Stamp $(T S)$ denotes the time that the message was generated. Finally, the field RV PHY Information carries the physical information of the RV.

\section{Algorithm of the VFHS Scheme}

Fig. 3 shows the detail of the VFHS algorithm. This algorithm runs in the OSV as well as in the BV, simultaneously. The first part, i.e., line 2-16, is to be run in the BV, and second part, line 17-42, is executed in the OSV. In the following, the set of channel frequencies and upper bound of the channel number are denoted $C$ and $C N_{\max }$, respectively. Assume that the OSV uses three channel frequencies to connect with BVs, i.e., $c_{i}, c_{j}, c_{k}$. Let $c_{i}, c_{j}, c_{k} \in C$, where $c_{i}, c_{j}, c_{k}$ denote channel frequencies that OSVs are using, and $0 \leq c_{i}, c_{j}, c_{k} \leq C N_{\max }$. An OSV chooses one of them to be its channel frequency when it disconnects with the RV. In the following, a symbol, such as $\mathbf{S}$, denotes the step of the algorithm, and other symbols, e.g. $\mathbf{S}$ ' and $\mathbf{S}$ ', mean two different sub-steps of the algorithm.

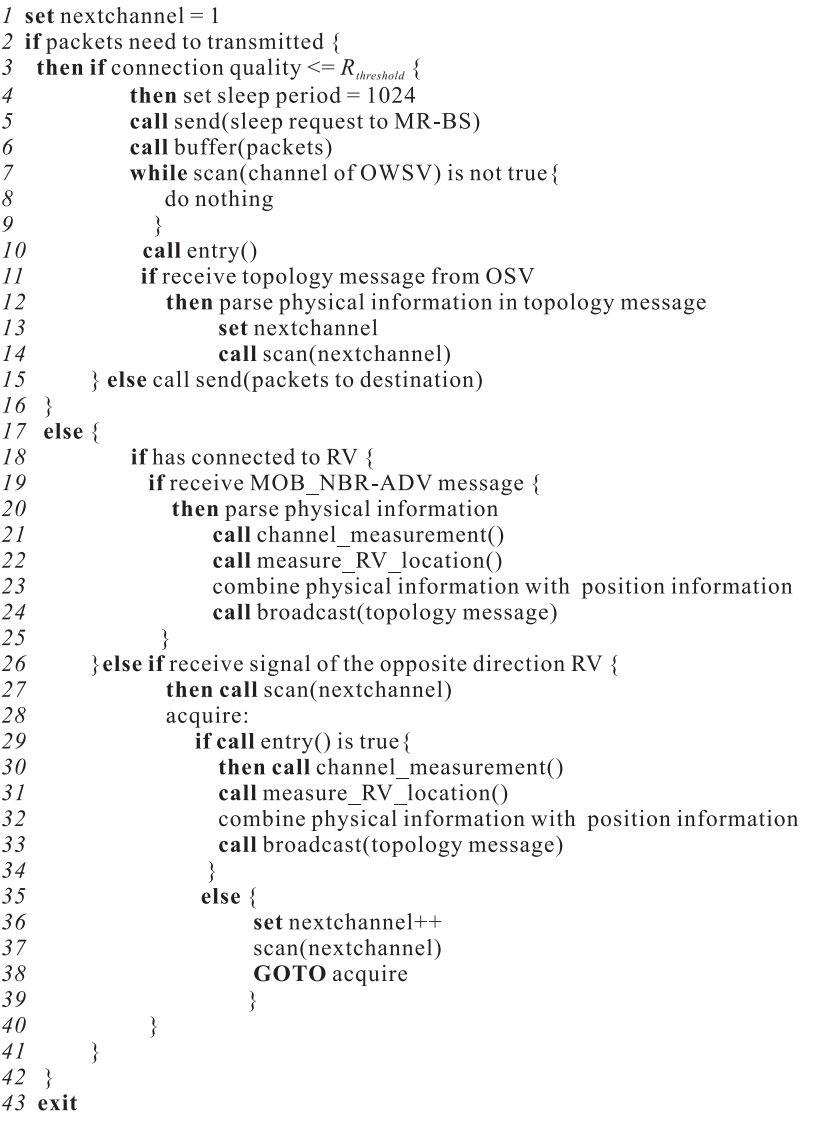

Fig. 3. Algorithm of VFHS scheme

At initiation phase, the vehicle classifies itself as a BV or an OSV. A vehicle acts as a BV if it maintains connection with a sender(s). Otherwise it becomes an OSV. Operation for a BV:

- S1: The BV detects the connection quality, $Q$, periodically by comparing the connection quality with a pre-defined threshold, $R_{\text {threshold }}$. The BV transmits a request for soliciting the RV to execute the sleep procedure, and begins to buffer packets when $Q \leq R_{\text {threshold }}$. Generally, the maximum period is 1024 frames, and can be terminated by force.

- S2: Upon receiving the sleep response message from the $\mathrm{RV}$, the BV at once selects one of $\left\{c_{i}, c_{j}, c_{k}\right\}$ to be its channel frequency, and performs pure entry procedure for connecting with the OSV.

- S3: Then, the BV will wait for acquiring the broadcasted topology message from the OSV. Finally, the BV scans the channel of the target RV because the physical layer information has been included in the topology message.

A vehicle becomes an OSV when is becomes disconnected and it can receive the signal of an oncoming RV. The algorithm then jumps to perform the OSV part. According to whether or not the OSV connects to the RV, the OSV part is divided into two subpart which are denoted $\mathbf{S}$ ' and $\mathbf{S}$ ', respectively.

The detail of the algorithm is described as follows.

- S1: The algorithm jumps to step S'1 since not linking to the RV. Or else the algorithm jumps to step $\mathbf{S}$ '1.

- S'1: The OSV receives the network advertisement message, MOB_NBR-ADV. The OSV then draws out the physical information of each of neighbor RVs from the MOB_NBR-ADV message.

- S'2: The OSV then sends channel measurement request message to each of neighbor RVs for detecting the RSSI and CINR information. According to RSSI and CINR, the OSV can execute other algorithm to predict the position of the RV. The algorithm jumps to step S2.

- S'1: In order to obtain the physical information, the OSV performs the pure entry procedure to connect to the RV for acquiring the network topology advertisement message which is broadcasted by the RV. Generally, the OSV should scan all channel frequency one by one or switch to an approximate channel frequency when it receives other network topology from the other OSV.

- S'2: After connecting to the RV, the OSV sends channel measurement request message to detect the RSSI and CINR information for predicting the position of the RV, similar to step S'2

- S2: Finally, the OSV integrates the physical information with position information, and broadcasts it to the BV using one of $\left\{c_{i}, c_{j}, c_{k}\right\}$. On the other hand, each of the topology message describes a specific RV. Therefore, the OSV may broadcast more than one topology messages since several RVs may appear at the same time. The BV prioritizes the order of the topology messages according to information of the RV position. The shorter distance between the RV and the BV should has higher priority.

\section{PERFormance AnAlysis}

\section{A. Simulation Environment}

To evaluate the VFHS scheme, we performed simulations using the network simulator NS-2 with NIST module [1]. 
Handover latency of VFHS is compared to that of the standard mobile WiMAX. Movement of vehicles is emulated using relative speed to RV's. For standing out the handover latency problem, the number of WiMAX channels is set to 30. Other parameters is the same as default values in the NIST module. Table I shows that other simulation parameters.

TABLE I

SYSTEM AND SCENARIO PARAMETERS

\begin{tabular}{|l|l|}
\hline \multicolumn{2}{|c|}{ System parameters } \\
\hline Modulation & OFDM_16QAM_3_4 $_{-}$162-13 \\
\hline RX Thresh & $1.26562 \mathrm{e}-13$ \\
\hline Packet size & 250 \\
\hline Scan Duration & 50 \\
\hline Interleaving interval & 40 \\
\hline Nbr_adv_interval & 30 \\
\hline T21timer & 0.5 \\
\hline DCD interval & 0.2 \\
\hline UCD interval & 0.2 \\
\hline contention size & 5 \\
\hline Association level & 0 \\
\hline
\end{tabular}

\section{B. Simulation scenario}

The goal of this simulation is to investigate and compare handover latency and packet loss of VFHS and that of standard WiMAX. Fig. 4 illustrates simulation topology used for the handover experiment. The performance metrics of interest are:

- Handover latency: The handover latency is defined as the time between when a BV is disconnected and when it resumes its connection with the new RV.

- Packet loss: The packet loss is the number of lost packets during handover.

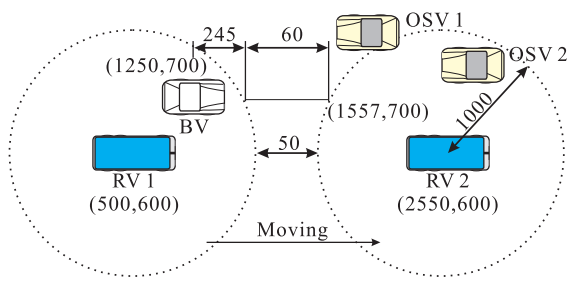

Fig. 4. Simulation Topology

1) Handoff Latency: Fig. 5 shows the handover latency under various relative speeds of a BV to $\mathrm{RV}$ when the $\mathrm{BV}$ handovers from RV 1 to RV 2. The handover latency shown is observed by a randomly picked BV. The distance between RV 1 and RV 2 is set to 2050 meters. The arrival process of OSV on the oncoming direction is assumed to be a Poisson process with arrival rate $\lambda$. From Fig. 5(a), we can observe that the handover latency decreases quickly as the relative speed of BV increases from 2 meter/second to 4 meter/second mainly because the time to pass through the disconnected area (gap) is reduced. In general, the handover procedure in standard WiMAX takes about 11 seconds. As the time for the $\mathrm{BV}$ to pass through the gap is less than 11 seconds, the handover latency is dominated by the handover procedure itself. Therefore, the increase of the relative speed does not
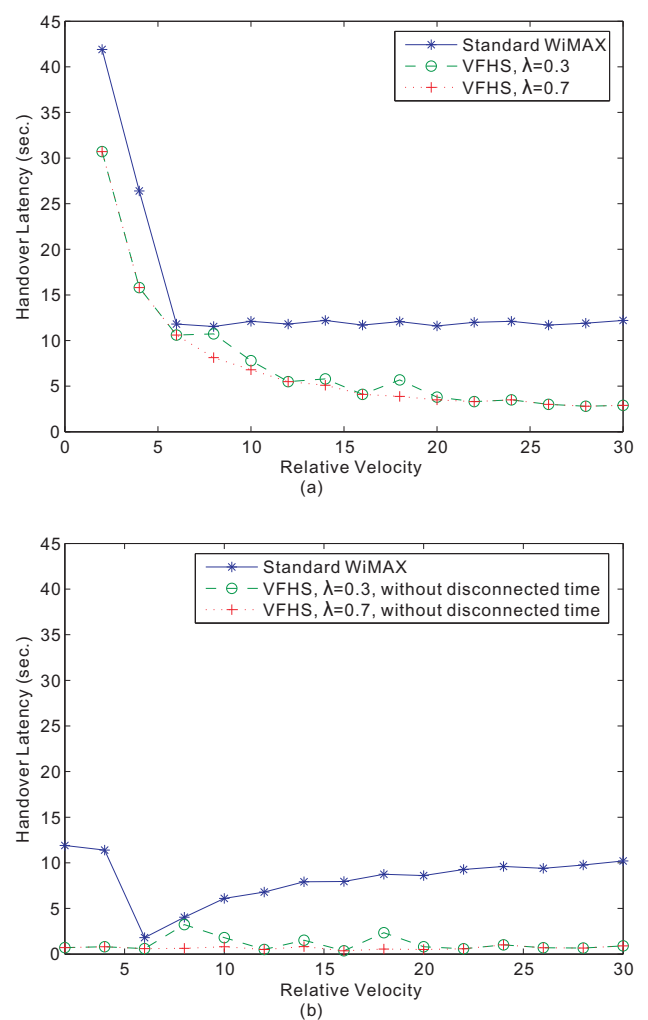

Fig. 5. Handover Latency under various relative speeds (a) handover latency (b)handover latency excluding the time to pass through the gap.

further decrease the handover latency. On the other hand, the VFHS scheme is able to reduce the handover latency as the $\mathrm{BV}$ can obtain the physical layer information of RV2 earlier. In addition, the time to pass through the gap is reduced as the speed of $\mathrm{BV}$ increases which continues to reduce the handover latency, as shown in Fig. 5(a). The arrival rate of OSV also affects the handover latency of VFHS, although not very significantly. The higher arrival rate, e.g., $\lambda=0.7$, the better chance that the $\mathrm{BV}$ receives the topology information broadcasted by an OSV.

Fig. 5(b) examines the handover latency excluding the time pass through the gap. For the standard WiMAX handover scheme, the handover latency only takes about 2 seconds if the BV scans to the right channel immediately after it enters the transmission range of RV2 which occurs when relative velocity is 6 meters/second. However, it could take up to 10 seconds to scan the to the right channel although it could pass through the gap at a very short time. The latency excluding the time pass through the gap is almost less than 2 seconds for the VFHS scheme no matter how fast is the relative speed. When the arrival rate of OSV is high, e.g., $\lambda=0.7$, the $\mathrm{BV}$ is quite likely to be able to receive the topology information from an OSV and the latency is quite stable (around 0.8 second). If the arrival rate is low, as we can see in Fig. 5(b), it may take a longer time to receive the topology information and thus increases the latency.

In Fig. 6, we further examine the effect of the OSV arrival 


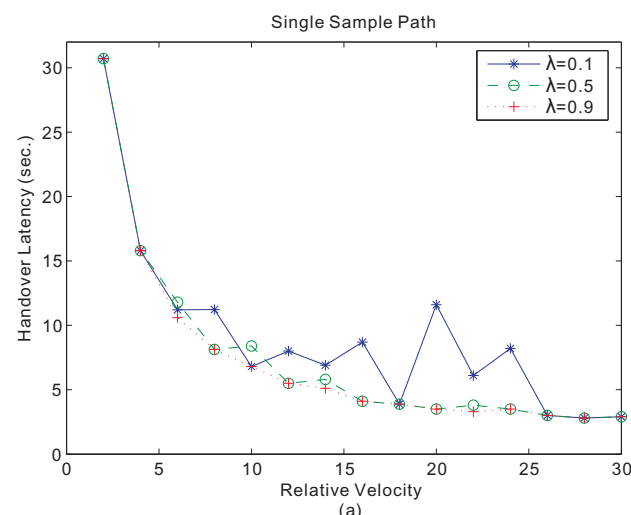

(a)

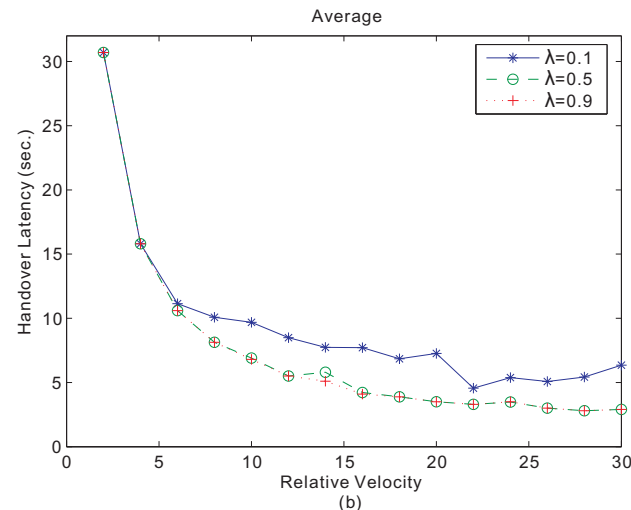

Fig. 6. Handover latency effect of OSV arrival rate on (a) single sample. (b) average.

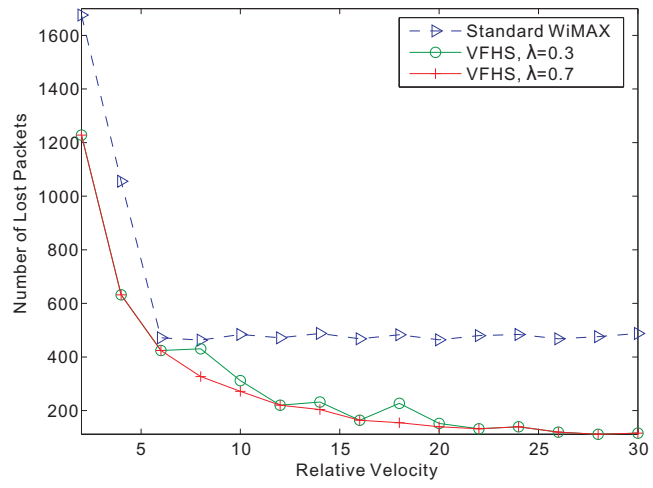

Fig. 7. Packet loss effect on relative velocity.

rate on the handover latency excluding the time pass through the gap. Fig. 6(a) shows the latency observed by a random BV while Fig. 6(b) shows the average latency. As we observed in Fig. 5, the higher the arrival rate, the lower the latency as the BV gets better chance to receive the topology information. Especially, when the arrival rate of OSV is low, the latency fluctuates significantly as shown in the case when $\lambda=0.1$ in Fig. 6. The additional delay is due to late appearing of an OSV.

2) Packet Loss: Figure 7 presents the number of lost packets during handover when the $\mathrm{BV}$ is receiving a constant bit rate stream. The numbers of lost packets under VFHS and WiMAX are compared at various relative speeds. Generally, the length of the disconnected period affects the number of lost packets. The VFHS resumes the connection faster than standard WiMAX handover. Additionally, if the arrival rate of OSV is higher, then the connection resumes more quickly. Therefore, because the VFHS has shorter handover latency than standard WiMAX, it also has fewer of lost packets. The higher arrival rate of OSV also further decreases the number of lost packets.

\section{CONClusion}

In this paper, we have proposed a context-aware fast handover scheme, called vehicular fast handover scheme (VFHS), for VANET formed on freeways. VFHS allows OSVs to provide physical layer information of an oncoming RV to oncoming BVs such that the handover latency could be significantly reduced. As the arrival rate of OSVs increases, a $\mathrm{BV}$ gets better chance to receive the physical layer information earlier and thus reduces its handover latency.

\section{ACKNOWLEDGMENTS}

The authors would like to thank the National Science Council of the Republic of China, Taiwan for financially supporting this research under Contract No. NSC97-2221-E194-027-MY2.

\section{REFERENCES}

[1] Ieee 802.16 module for ns-2.

[2] Publication.

[3] P. Boone, M. Barbeau, and E. Kranakis. Strategies for fast scanning and handovers in WiMAX/802.16. In Proc. Second International Conference on Access Networks \& Workshops AccessNets '07, pages 1-7, 22-24 Aug. 2007.

[4] J. Chen, C.-C. Wang, and J.-D. Lee. Pre-Coordination Mechanism for Fast Handover in WiMAX Networks. In Proc. 2nd International Conference on Wireless Broadband and Ultra Wideband Communications AusWireless 2007, pages 15-15, 27-30 Aug. 2007.

[5] L. Chen, X. Cai, R. Sofia, and Z. Huang. A Cross-Layer Fast Handover Scheme For Mobile WiMAX. In Proc. VTC-2007 Fall Vehicular Technology Conference 2007 IEEE 66th, pages 1578-1582, Sept. 30 2007-Oct. 32007.

[6] R.-H. Hwang and K.-L. Chiu. Communication Framework for Vehicle Ad Hoc Networks on Freeways. in Preparation.

[7] M. Jerbi and S. M. Senouci. Characterizing Multi-Hop Communication in Vehicular Networks. In Proc. IEEE Wireless Communications and Networking Conference WCNC 2008, pages 3309-3313, March 31 2008-April 32008.

[8] S. Kota, G. Giambene, and N. L. Candio. Cross-layer approach for an air interface of geo satellite communication networks. International Journal of Satellite Communications and Networking, 25(5):481-499, 2007.

[9] J. H. Park, K.-Y. Han, and D.-H. Cho. Reducing Inter-Cell Handover Events based on Cell ID Information in Multi-hop Relay Systems. In Proc. VTC2007-Spring Vehicular Technology Conference IEEE 65th, pages 743-747, 22-25 April 2007.

[10] R. Rouil and N. Golmie. Adaptive Channel Scanning For IEEE 802.16e. In Proc. Military Communications MILCOM 2006, pages 1-6, 23-25 October 2006.

[11] H. Saleet and O. Basir. Location-Based Message Aggregation in Vehicular Ad Hoc Networks. In Proc. IEEE Globecom Workshops, pages 1-7, 26-30 Nov. 2007.

[12] V. Srivastava and M. Motani. Cross-layer design: a survey and the road ahead. 43(12):112-119, 2005. 